\section{Detection of Novel}

\section{Coronavirus by RT-PCR in Stool Specimen from Asymptomatic Child, China}

\author{
An Tang, ${ }^{1}$ Zhen-dong Tong, ${ }^{1}$ Hong-ling Wang, ${ }^{1}$ \\ Ya-xin Dai, ${ }^{1}$ Ke-feng Li, Jie-nan Liu, Wen-jie Wu, \\ Chen Yuan, Meng-lu Yu, Peng Li, Jian-bo Yan \\ Author affiliation: Zhoushan Center for Disease Control and \\ Prevention, Zhoushan, China
}

DOI: https://doi.org/10.3201/eid2606.200301

We report an asymptomatic child who was positive for a coronavirus by reverse transcription PCR in a stool specimen 17 days after the last virus exposure. The child was virus positive in stool specimens for at least an additional 9 days. Respiratory tract specimens were negative by reverse transcription PCR.

A n outbreak of coronavirus disease (COVID-19) began in Wuhan, China, during December 2019 and has rapidly spread throughout China and to many countries $(1,2)$. Common symptoms include fever, dry cough, and myalgia (3). Ten laboratoryconfirmed cases and several asymptomatic cases of COVID-19 have been identified in Zhoushan, China, since January 19, 2020. We report the epidemiologic and diagnostic features for 1 case in an asymptomatic child.

On January 30, 2020, we identified a 10-year-old boy who had no fever or cough but had close contact with 2 confirmed case-patients with laboratory-confirmed COVID-19 (Figure). The boy was a primary school student who lived with his parents in an apartment of a college. The complex had several confirmed COVID-19 case-patients during January 19-31.

Interviews of the boy and his parents confirmed his multiple exposures to the previously confirmed case-patients. On January 9 and 15, he participated in 2 parties with his parents and their colleagues. Two persons at these parties were positive for severe acute respiratory syndrome coronavirus 2 (SARS-CoV-2) by reverse transcription PCR (RT-PCR) on January 20 and 22. During January 12-15, the boy played football at a football club with a teammate who had a viruspositive RT-PCR result on January 22. The parents of the boy were asymptomatic and their stool, nasopharyngeal, and sputum specimens collected on February 1 and 14 were negative for SARS-CoV-2.

${ }^{1}$ These authors contributed equally to this article.
We collected nasopharyngeal swab and sputum samples from the boy 15 days after the last close contact and tested these specimens for SARS-CoV-2 by using RT-PCRs targeting the open reading frame lab (ORF1ab) and nucleoprotein gene regions (4). We obtained equivocal results: cycle threshold $\left(C_{t}\right)$ values were negative for ORFlab and 37.5 for the nucleoprotein gene. However, on February 1 (17 days after his last contact), a stool specimen was positive for SARSCoV-2 by RT-PCR. (ORF1ab $C_{t} 32.6$; nucleoprotein gene $C_{t}$ 33.7). He was then hospitalized in isolation and for monitoring.

Since January 22, The area of residence for the boy had been isolated, and community physicians monitored quarantined residents twice a day for signs and symptoms including fever, cough, and myalgia. During January 9-31, the boy had no signs or symptoms.

In the hospital, a routine blood test performed on February 2 showed cell counts within reference ranges, and a computed tomography scan on February 5 showed no abnormalities. After additional stool specimens collected on February 2 (ORF1ab C 25.6; nucleoprotein gene $C_{t} 25.8$ ) and February 4 (ORF1ab $C_{t}$ 25.6; nucleoprotein gene $C_{t} 28.3$ ) were positive, the patient received abidol hydrochloride $(100 \mathrm{mg} 3 \times / \mathrm{d})$, interferon $\mathrm{a}-2 \mathrm{~b}$ spray $(2.5$ million $\mathrm{U} 2 \times / \mathrm{d})$ and traditional Chinese medical therapy on February 5. Stool specimens collected on February 7 (ORF1ab C $C_{t} 26.3$; nucleoprotein gene $C_{t}$ 27.6), February 8 (ORF1ab $C_{t}$ 31.4; nucleoprotein gene $C_{t} 30.6$ ), and February 9 (ORF1ab $C_{t}$ 27.0; nucleoprotein gene $C_{t} 27.0$ ) were positive, but stool specimens collected on February 12 and 14 were negative.

Early symptoms in most COVID-19 patients include fever, myalgia, cough, and sore throat (5), which are common in other acute respiratory virus infections (6). Most cases appear to be mild, and most hospitalized patients have pneumonia with ground glass opacities on chest radiographs. Few children with SARS-CoV-2 infections have been reported, and most of them had mild clinical symptoms (7).

The boy we report had close contact with confirmed COVID-19 case-patients on several occasions before he showed an equivocal RT-PCR result for respiratory specimens and subsequently positive results for stool specimens. Despite these positive test results, he had no detectable fever or other clinical symptoms consistent with COVID-19 for $\geq 30$ days from his last documented exposure. Although positive RT-PCR results do not necessarily indicate presence of infectious virus, our findings reinforce the need for RT-PCR testing of asymptomatic persons with exposure to COVID-19 patients. 


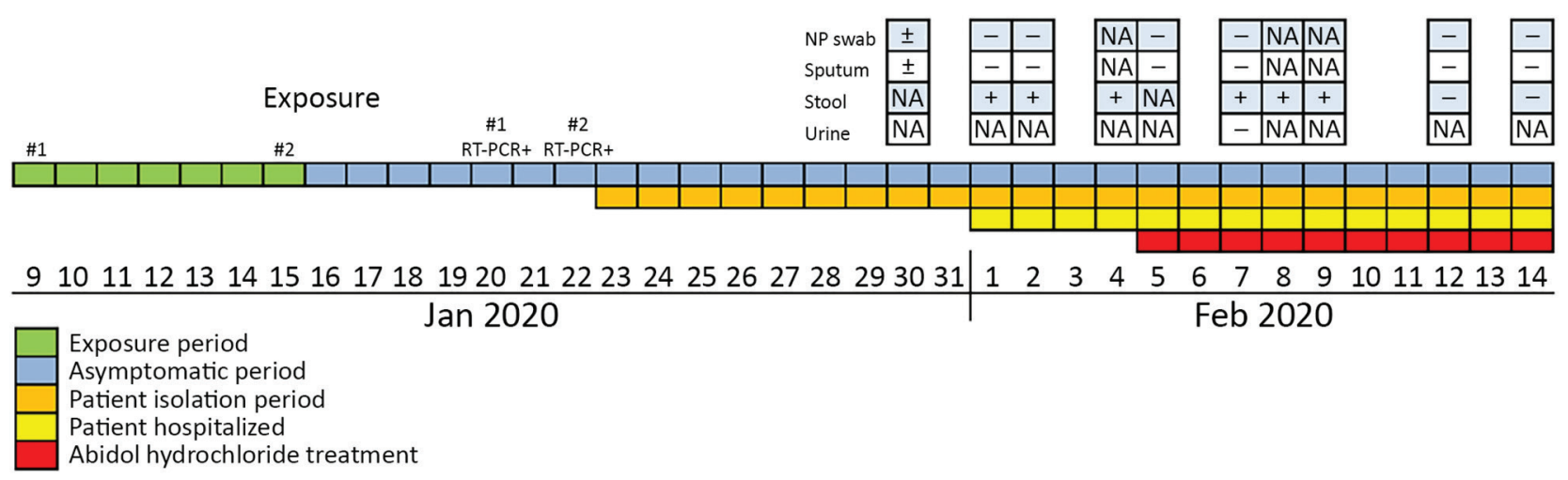

Figure. Timeline for detection of novel coronavirus by RT-PCR in stool specimen from asymptomatic child, China, January 9-February 14, 2020. NA, not available; NP, nasopharyngeal; RT-PCR, reverse transcription PCR; +, positive for novel coronavirus RNA by RTPCR; \pm , equivocal for novel coronavirus RNA by RT-PCR; -, negative for novel coronavirus RNA by RT-PCR.

Asymptomatic infections complicate efforts to curtail SARS-CoV-2 transmission and implement effective control procedures.

SARS-CoV-2 is believed to be transmitted through large respiratory droplets (8) and close contact (9). Indirect transmission by contaminated fomites might also play a role. During the SARS pandemic of 2002-2003, positive RT-PCR results for stool specimens from SARS patients suggested that stools or sewage might be virus sources (10). Our finding of multiple positive stool specimens in this case similarly raises the concern that stool from COVID-19 patients might serve as another vehicle for virus transmission. Moreover, detection of virus by RT-PCR in stool specimens when respiratory tract specimens are negative suggests that stool might be considered, in addition to respiratory tract specimens, for routine diagnostic screening.

Our study had several limitations. The delay in RT-PCR testing after the first recognition of virus exposure prevented a more accurate estimation of the incubation time from exposure to RT-PCR positivity. The failure to test other specimens, such as blood and urine, prevented determination of the full spectrum of virus shedding for the case-patient. Although we urge caution in making policy decisions on the basis of 1 case, expanded testing of various clinical specimens from symptomatic and asymptomatic case-patient contacts at multiple time points would be warranted to help confirm our findings.

\section{Acknowledgment}

We thank all members of the Zhoushan Center for Disease Control and Prevention for their involvement during the response to the outbreak.
This study was supported by the Zhoushan Science And Technology Project (grant nos. 2020C31004, 2020C31005, and 2020C31006); the Zhejiang Scientific and

Technological Major Project under the 2020 Emergency (grant no. 2020C03124); the Zhejiang University special scientific research fund for COVID-19 prevention and control; and the Zhejiang Natural Project on Emergency Research about Community Prevention, Control, Early Warning, and Prediction of the novel coronavirus outbreak (grant no. LEZ20H260001)

\section{About the Author}

Dr. Tang is a public health research scientist at the Zhoushan Center for Disease Control and Prevention, Zhoushan, China. His research interests are epidemiology and control of infectious diseases.

\section{References}

1. Xiang YT, Yang Y, Li W, Zhang L, Zhang Q, Cheung T, et al. Timely mental health care for the 2019 novel coronavirus outbreak is urgently needed. Lancet Psychiatry. 2020;7:228-9 [Epub ahead of print]. https:/ / doi.org/10.1016/ S2215-0366(20)30046-8

2. Holshue ML, DeBolt C, Lindquist S, Lofy KH, Wiesman J, Bruce H, et al.; Washington State 2019-nCoV Case Investigation Team. First case of 2019 novel coronavirus in the United States. N Engl J Med. 2020;382:929-36. https://doi.org/10.1056/NEJMoa2001191

3. Wang D, Hu B, Hu C, Zhu F, Liu X, Zhang J, et al. Clinical characteristics of 138 hospitalized patients with 2019 novel coronavirus-infected pneumonia in Wuhan, China. JAMA. 2020 Feb 7 [Epub ahead of print]. https://doi.org/10.1001/ jama.2020.1585

4. Zhu N, Zhang D, Wang W, Li X, Yang B, Song J, et al.; China Novel Coronavirus Investigating and Research Team. A novel coronavirus from patients with pneumonia in China, 2019. N Engl J Med. 2020;382:727-33. https://doi.org/10.1056/NEJMoa2001017

5. Chen N, Zhou M, Dong X, Qu J, Gong F, Han Y, et al. Epidemiological and clinical characteristics of 99 cases of 
2019 novel coronavirus pneumonia in Wuhan, China: a descriptive study. Lancet. 2020;395:507-13. https://doi.org/ 10.1016/S0140-6736(20)30211-7

6. Kim JY, Choe PG, Oh Y, Oh KJ, Kim J, Park SJ, et al. The first case of 2019 novel coronavirus pneumonia imported into Korea from Wuhan, China: implication for infection prevention and control measures. J Korean Med Sci. 2020;35:e61. https:/ / doi.org/10.3346/ jkms.2020.35.e61

7. Del Rio C, Malani PN. Novel coronavirus-important information for clinicians. JAMA. 2020 Feb 5 [Epub ahead of print]. https://doi.org/10.1001/jama.2020.1490

8. Kampf G, Todt D, Pfaender S, Steinmann E. Persistence of coronaviruses on inanimate surfaces and their inactivation with biocidal agents. J Hosp Infect. 2020;104:246-51. https:/ / doi.org/10.1016/j.jhin.2020.01.022

9. To KK, Tsang OT, Chik-Yan Yip C, Chan KH, Wu TC, Chan JMC, et al. Consistent detection of 2019 novel coronavirus in saliva. Clin Infect Dis. 2020 Feb 12 [Epub ahead of print] https://doi.org/10.1093/cid/ciaa149

10. Wang XW, Li JS, Guo TK, Zhen B, Kong QX, Yi B, et al. Concentration and detection of SARS coronavirus in sewage from Xiao Tang Shan Hospital and the 309th Hospital. J Virol Methods. 2005;128:156-61.

https://doi.org/10.1016/j.jviromet.2005.03.022

Address for correspondence: Ya-xin Dai or Jian-bo Yan, Zhoushan Center for Disease Control and Prevention, No. 568 Wengshan Rd, Zhejiang, Zhoushan 316021, China: email: daiyaxin.712@163.com or yanjianbo02@163.com

\section{Case-Fatality Risk Estimates for COVID-19 Calculated by Using a Lag Time for Fatality}

\author{
Nick Wilson, Amanda Kvalsvig, Lucy Telfar Barnard, \\ Michael G. Baker
}

Author affiliations: University of Otago Department of Public Health, Wellington, New Zealand

DOI: https://doi.org/10.3201/eid2606.200320

We estimated the case-fatality risk for coronavirus disease cases in China (3.5\%); China, excluding Hubei Province $(0.8 \%) ; 82$ countries, territories, and areas $(4.2 \%)$; and on a cruise ship $(0.6 \%)$. Lower estimates might be closest to the true value, but a broad range of $0.25 \%-3.0 \%$ probably should be considered.
7 he coronavirus disease (COVID-19) is spreading 1 globally; as of March 5, 2020, cases were reported in China and 85 other countries, territories, and areas (1). Disease severity is a particularly crucial parameter for understanding this new disease (2), but accurately estimating the case-fatality risk is difficult because milder cases are not being diagnosed and death is delayed.

We used data from the World Health Organization (WHO) (1) to calculate crude estimates of the casefatality risk on March 5, 2020, for 4 populations: China; China, excluding Hubei Province; a group of 82 countries, territories, and areas; and passengers and crew of a cruise ship (Table 1). However, given the critical need to consider time lags to death when calculating casefatality risk (3), we used time lags from a recent study from China (4). Yang et al. (4) reported that the median time from symptom onset to radiological confirmation of pneumonia was 5 days (interquartile range [IQR] 3-7 days); from symptom onset to intensive care unit (ICU) admission was 11 days (IQR 7-14 days); and from ICU admission to death was 7 days (IQR 3-11 days). Therefore, a median of 13 days passed from pneumonia confirmation to death $([11-5]+7=13)$.

For our calculation, we assumed that the day of radiological confirmation of pneumonia approximately equated to the reporting date for laboratoryconfirmed cases of COVID-19 to WHO. We obtained cumulative COVID-19 case counts reported by WHO on February 21 (5), which was 13 days before March 5 , the date we used for calculating the crude case-fatality risk. Our approach is broadly comparable to a study that used earlier data to estimate the median time delay of 13 days from illness onset to death (6).

By using the number of cumulative cases on February 21 as the denominator for the adjusted case-fatality risk (aCFR), we assumed that half of the additional cumulative reported deaths on March 5 could be matched with cases reported on February 21. We acknowledge our approach is fairly simplistic and that it can be superseded when higher quality cohortbased analyses become available.

The case-fatality risks, when adjusted for a 13day lag time from reporting to death, were 3.5\% in China; $0.8 \%$ in China, excluding Hubei Province; $4.2 \%$ in the group of 82 countries, territories, and areas; and $0.6 \%$ for the cruise ship (Table). Our result for China, excluding Hubei Province, is similar to a previous estimate of $0.9 \%$ (95\% CI $0.6 \%-1.3 \%)$ by using a time-delay adjusted case-fatality risk for the same area (K. Mizumoto and G. Chowell, unpub. data; https://www.medrxiv.org/content/10.1101/ 2020.02.19.20025163v1). 